\title{
Ingenierías de aditivos en celdas solares tipo perovskita
}

\author{
Ana Itzel Santiago Mustafat ${ }^{A}$, Arián Espinosa Roa ${ }^{B}$ Edgar \\ González Juárez ${ }^{A}$, Eduardo M. Sánchez Cervantes ${ }^{A^{*}}$ \\ AUniversidad Autónoma de Nuevo León, Facultad de Ciencias Químicas \\ ${ }^{\mathrm{B} C e n t r o ~ d e ~ I n v e s t i g a c i o ́ n ~ e n ~ Q u i ́ m i c a ~ A p l i c a d a, ~ C I Q A ~}$ \\ *eduardo.sanchezcv@uanl.edu.mx
}

\section{RESUMEN}

Las celdas solares de perovskita (CSP) han cambiado el paradigma de investigación en el área de la energía fotovoltaica debido a la combinación de altas eficiencias junto con un menor costo y facilidad de fabricación. Las CSP se pueden fabricar mediante metodologías basadas en soluciones de compuestos precursores para el depósito de las películas de estructura tipo perovskita. Entre esos compuestos se encuentran los haluros de plomo inorgánicos ( $\mathrm{Pbl}_{2}$, $\left.\mathrm{PbCl}_{2}, \mathrm{PbBr}_{2}\right)$ en combinación con compuesto orgánico metilamonio (MA) que se reporta que han alcanzado hasta un $25 \%$ de eficiencia. A pesar de ello, estos materiales presentan desventajas como la sensibilidad de la película de perovskita a la humedad del ambiente, lo que resulta un tiempo de vida de los dispositivos corto. Una alternativa para estabilizar la celda es la aplicación de aditivos, los cuales son líquidos iónicos formados por un catión y un anión con un carácter altamente hidrófobo, basados en fosfonio (tetraburoborato de tetrabutil fosfonio $\left(B_{4} P B F_{4}\right)$. El aditivo mejora significativamente la morfología de las películas, obteniendo mejoras prometedoras en la estabilidad de los dispositivos.

\section{PALABRAS CLAVE}

Celdas solares, perovskitas, líquidos iónicos, capas delgadas, estabilidad.

\section{ABSTRACT}

Perovskite (CSP) solar cells have changed the research paradigm in the area of photovoltaics, due to the combination of high efficiencies along with lower cost and ease of manufacture. CSP can be manufactured using methodologies based on solutions of precursor compounds for the deposition of perovskite films. Among these compounds are the inorganic lead halides $\left(\mathrm{Pbl}_{2}, \mathrm{PbCl}_{2}\right.$, $\left.\mathrm{PbBr}_{2}\right)$ in combination with organic methylammonium (MA), with reported efficiency values up to $25 \%$. Despite their high efficiencies, these materials have disadvantages, such as the sensitivity of the perovskite film to ambient humidity, resulting in a short device life time. An alternative to reduce stability problems is the application of additives that increase the stability of the cell. Said additives are ionic liquids formed by a cation and an anion with a highly hydrophobic character, based on phosphonium (tetrabutyl phosphonium tetraburoborate $\left(B_{4} P B F_{4}\right)$. The additive significantly improves the morphology of the films, obtaining promising improvements in the stability of the devices.

\section{KEYWORDS}

Solar cells, perovskites, ionic liquids, thin films, stability. 


\section{INTRODUCCIÓN}

La energía solar es una fuente de energía limpia que puede ayudar a satisfacer la creciente demanda mundial de energía, así como disminuir los efectos de emisión de carbono. Entre los dispositivos que proveen este tipo de energía se encuentran las celdas solares de perovskita (CSP). En los últimos años ha crecido su relevancia debido a las altas eficiencias de conversión (PSC) reportadas a más del $25 \%,{ }^{1}$ sumando a su alto nivel de absorción y un menor costo de fabricación. Componentes diversos en la solución precursora de la perovskita de haluro organometálico $\mathrm{CH}_{3} \mathrm{NH}_{3} \mathrm{MX}_{3}(\mathrm{M}=\mathrm{Pb}$ o $\mathrm{Sn}, \mathrm{X}=\mathrm{Cl}$, Bro I), afectan fuertemente la calidad de la película cristalina de la perovskita, formando una variedad de defectos. Las perovskitas de haluro organometálico, se reportaron por primera vez en 1970 con una fórmula general $\mathrm{ABX}_{3}$, donde $\mathrm{A}^{+}=$un catión orgánico como metilamonio $\left(\mathrm{CH}_{3} \mathrm{NH}_{3}{ }^{+}\right.$y MA $\left.{ }^{+}\right)$, cesio $\left(\mathrm{Cs}^{+}\right)$, rubidio $\left(\mathrm{Ru}^{+}\right)$, formamidinio $(\mathrm{HC}$ $\left(\mathrm{NH}_{2}\right)_{2}$ y FA $\left.{ }^{+}\right) ; \mathrm{B}=$ plomo $\left(\mathrm{Pb}_{2}^{+}\right)$o estaño $\left(\mathrm{Sn}_{2}^{+}\right)$y X- = cloro $\left(\mathrm{Cl}^{-}\right)$, bromo $(\mathrm{Br})$ o yodo $\left(1^{-}\right){ }^{2}$ En 1995 Mitzi et al., desarrollo a profundidad el procesamiento de cristales de perovskita orgánicos e inorgánicos en capas y sus propiedades ópticas. ${ }^{3}$ Las celdas solares de perovskita basadas en haluro organometálico, como capa absorbente de luz, se han considerado prometedoras en la tecnología fotovoltaica. El material clave que ha presentado estas eficiencias es el haluro organometálico $\mathrm{CH}_{3} \mathrm{NH}_{3} \mathrm{MX}_{3}(\mathrm{M}=\mathrm{Pb}$ o $\mathrm{Sn}, \mathrm{X}=\mathrm{Cl}, \mathrm{Br}$ o I $)$. Componentes diversos en la solución precursora de la perovskita de haluro organometálico afectan fuertemente la calidad de la película cristalina de la perovskita, formando una variedad de defectos. Actualmente, los aditivos se utilizan ampliamente, generando mejoras significativas en las CSP. Son et al. en 2018, demostraron que el yoduro de potasio (KI) en perovskita de haluro, puede eliminar la histéresis de los dispositivos alcanzando eficiencias alrededor del $17 \% .{ }^{4}$ Ésta y otras investigaciones como Zhang et al. en 2019 sugieren que el yoduro de potasio (KI) y el triyoduro de potasio $\left(\mathrm{KI}_{3}\right)$ son un ejemplo de aditivos inorgánicos útiles. Que modifican la morfología de la superficie de la película de perovskita, mejorando la estabilidad del dispositivo con un $21 \%$ de PCE. ${ }^{5,6}$ Los líquidos iónicos (LI) también han sido utilizados como disolventes y/o aditivos en soluciones precursoras de perovskita, así como modificadores de interfaz. Debido a la naturaleza de los $\mathrm{LI}$, los precursores $\left(\mathrm{Pbl}_{2}\right.$ y $\left.\mathrm{MAI}\right)$, se disuelven homogéneamente en la solución, cuando se usan como solvente o aditivo promueven la formación uniforme de la película. ${ }^{7,8}$ Los LI son ampliamente utilizados para estabilizar los dispositivos contra la humedad. En una publicación de Du et al. 2018, donde utilizan 1-butil-3metilimidazolio (BMIBr) como aditivo en la solución precursora de la perovskita $\left(\mathrm{MAPbI}_{3}\right)$, las películas dopadas mostraron tolerar mayormente la estabilidad térmica $\left(85^{\circ} \mathrm{C}\right.$ por 50 minutos), en contraste de las películas no dopadas $\left(85^{\circ} \mathrm{C}\right.$ por 20 minutos), este enfoque puede ayudar a desarrollar dispositivos fotovoltaicos a largo plazo. ${ }^{9}$ La naturaleza hidrófoba de los LI también han sido altamente efectiva para mejorar la estabilidad de las CSP, estos están siendo explorados recientemente. ${ }^{9}$ La mayoría de las investigaciones de los LI se centran en los basados en nitrógeno (amonio, imidazolio, piridinio), mientras que los LI basados en fósforo han recibido menos atención, a pesar de las propiedades comparables

a sus contrapartes a base de nitrógeno. Los fosfonios son compuestos orgánicos ampliamente reconocidos como hidrófobos y estables contra la radiación solar. ${ }^{10}$ 
Varios haluros de fenilfosfonio han sido utilizados ${ }^{11}$ como aditivos, tales como el yoduro de tetrafenilfosfonio (TPPI), cloruro de tetrafenilfosfonio (TPPCl) y bromuro de tetrafenilfosfonio (TPPBr) en la que reportaron que el TPPI y TPPCl son eficientes en mejorar la cristalinidad y coberturas de las películas de perovskita (figura 2), esto se debe a que los aniones 1- y Cl- pueden quelatarse con los cationes del $\mathrm{Pb}_{2}{ }^{+}$y mejorar la formación de la película. Como resultado obtuvieron una eficiencia mejorada que va de un $10 \%$ para la celda solar de referencia sin aditivos a un $13 \%$ con aditivo interfacial. ${ }^{12}$ Por otro lado, Wu et al. reporta que utilizaron el aditivo 1- hexafluorofosfato de etil-3-metilimidazolio ([EMIM]PF ${ }_{6}$ ) entre la capa ETL y la capa activa. Como resultado obtuvieron una mejora de transporte de electrones, reflejada en su alta eficiencia de conversión de energía del $19.5 \% .^{13}$
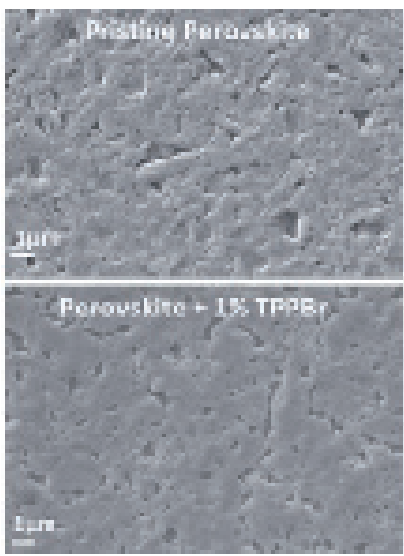
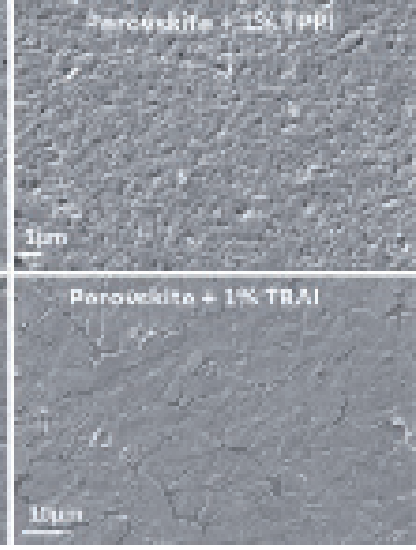

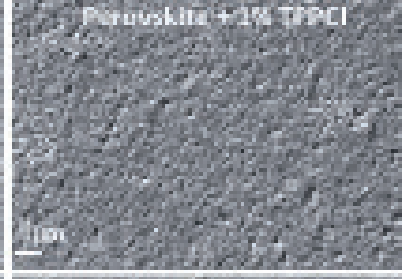

Peravikitei is itap

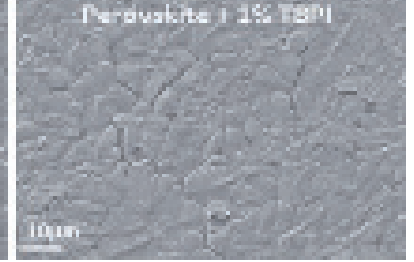

Fig. 1. Micrografías SEM de películas TPPCI, TBPI, TPPI, TPPBr y TBAl (tomada de Phosphonium Halides as Both Processing Additives and Interfacial Modifiers for High Performance Planar- Heterojunction Perovskite Solar Cells, Small, Sun, 2015). ${ }^{12}$

En el laboratorio de Materiales para el Almacenamiento y Conversión de Energía de la División de Estudios de Posgrado de la Facultad de Ciencias Químicas (FCQ), de la Universidad Autónoma de Nuevo León (UANL), se está llevando a cabo un estudio de la síntesis de un nuevo líquido iónico (LI) para incorporarlo en los precursores $\left(\mathrm{Pbl}_{2}\right.$ y $\left.\mathrm{MAI}\right)$ de perovskitas híbridas, para un estudio más amplio el aditivo se incorporó en diversos intervalos $(0 \%, 0.25 \%$, $0.75 \%$ y $1.5 \%$ ) para mejorar su estabilidad. En el presente reporte se describen los métodos experimentales iniciales de la síntesis de un nuevo líquido iónico tetraburoborato de tetrabutil fosfonio $\left(\mathrm{B}_{4} \mathrm{PBF}_{4}\right)$ y sus resultados obtenidos para la integración del LI dentro de la CSP. Integrando el aditivo $\mathrm{B}_{4} \mathrm{PBF}_{4}$ en la película activa perovskita en la solución precursora.

\section{EXPERIMENTAL}

\section{Síntesis de yoduro de tetrabutil fosfonio (B4PI)}

La síntesis se llevó a cabo dentro de la caja de guantes debido a que el tributilfosfonio es muy reactivo. Se comenzó pesando en una botella ámbar $2.667 \mathrm{~g}$ de ioduro de butilo y $3.018 \mathrm{~g}$ de tributilfosfonio en una relación estequiométrica de 1:1. La mezcla se zonifico durante 6 horas, fuera de la cámara seca. Pasado dicho tiempo se obtuvo un sólido blanco, el cual se le dieron un periodo de 
Ingenierías de aditivos en celdas solares tipo perovskita / Ana Itzel Santiago Mustafat, et al.

10 lavados con éter de petróleo por 10 minutos cada lavado en vortex para su purificación.

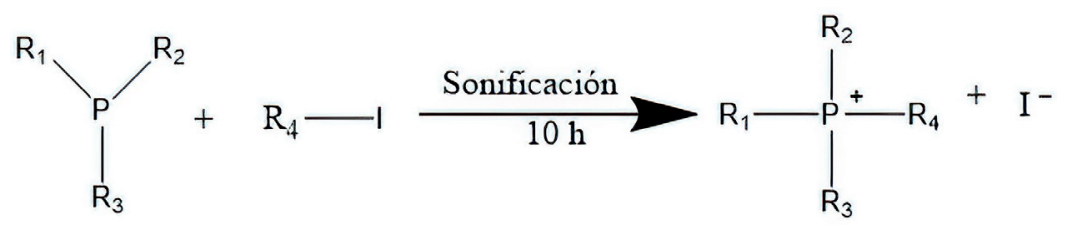

\section{Síntesis del líquido iónico utilizando agua como disolvente}

Se comenzó pesando en un frasco ámbar $485 \mathrm{mg}$ de KBF4 se disolvió en agua y posterior se añadió $500 \mathrm{mg}$ de B4PI en, en una relación de 1:2 se disolvieron en $10 \mathrm{~mL}$ de agua. La reacción se precipitó por medio de sonificación durante 10 horas. El precipitado formado se filtró y se lavó con agua para descartar algún residuo de KI. El precipitado final se pasó al horno por unos minutos para evaporar el agua.

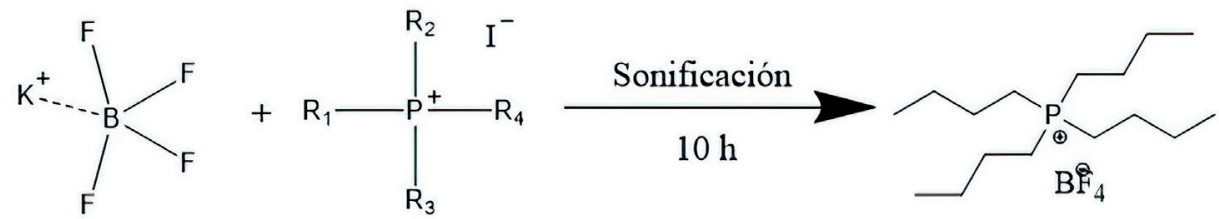

\section{Incorporación de $\mathrm{B}_{4} \mathrm{PBF}_{4}$ sintetizado en la película $\mathrm{MAPbI}_{3}$}

Por el método de dos pasos se preparo una solución de $\mathrm{PbI}_{2}$ y MAI en proporción molar $1: 1$, con una concentración $1.25 \mathrm{M}$. Se disolvió primero el yoduro de plomo en una mezcla 4:1 en volumen de DMF y DMSO, la solución se agitó durante dos horas a $60^{\circ} \mathrm{C}$ antes de agregar la cantidad correspondiente de MAI y $\mathrm{B}_{4} \mathrm{PBF}_{4}$ a cada una de las soluciones, resumidas en la tabla I. Las películas se depositaron mediante spin coating $5000 \mathrm{rpm}$ a $30 \mathrm{~s}$ utilizando clorobenceno como antisolvente a los seis segundos de iniciado el spin coating. Seguido de un tratamiento térmico durante 10 minutos a $100{ }^{\circ} \mathrm{C}$.

Tabla I. Diferentes composiciones utilizadas en las síntesis de soluciones derivadas de $\mathrm{MAPbl}_{3}-\mathrm{B}_{4} \mathrm{PBF}_{4}$.

\begin{tabular}{|l|c|c|c|}
\hline \multicolumn{1}{|c|}{ Solución } & $\mathrm{Pbl}_{2}(\mathrm{mg})$ & $\mathrm{CH}_{3} \mathrm{NH}_{3} \mathrm{l}(\mathrm{mg})$ & $\mathrm{B}_{4} \mathrm{PBF}_{4}$ \\
\hline $\mathrm{MAPbl}_{3}$ & $289 \mathrm{mg}$ & $100 \mathrm{mg}$ & $0 \%$ \\
\hline$M A 99.75\left(\mathrm{~B}_{4} \mathrm{PBF}_{4}\right) 0.25 \mathrm{Pbl}_{3}$ & $289 \mathrm{mg}$ & 99.75 & $0.25 \%$ \\
\hline$M A 99.25\left(\mathrm{~B}_{4} \mathrm{PBF}_{4}\right) 0.75 \mathrm{Pbl}_{3}$ & $289 \mathrm{mg}$ & 99.25 & $0.75 \%$ \\
\hline$M A 98.5\left(\mathrm{~B}_{4} \mathrm{PBF}_{4}\right) 1.5 \mathrm{Pbl}_{3}$ & $289 \mathrm{mg}$ & 98.5 & $1.5 \%$ \\
\hline
\end{tabular}

\section{RESULTADOS}

Para determinar la obtención del $\mathrm{LI}-\mathrm{B}_{4} \mathrm{PBF}_{4}$, se utilizó como técnica espectroscopia infrarroja (FTIR) y Resonancia magnética nuclear (RMN) de $\mathrm{H}^{1}, \mathrm{P}^{31}, \mathrm{~B}^{11}$. En la figura 2a, donde se compararon los espectros de las bandas significativas de los compuestos de los materiales de partida, reflejados en el espectro azul de $\mathrm{B}_{4} \mathrm{PBF}_{4}$. En la figura $2 \mathrm{~b}$ ), se muestra el análisis de espectroscopia $\mathrm{UV}-\mathrm{V}$ is de las películas con la incorporación de $\mathrm{B}_{4} \mathrm{PBF}_{4}$, donde muestran los 

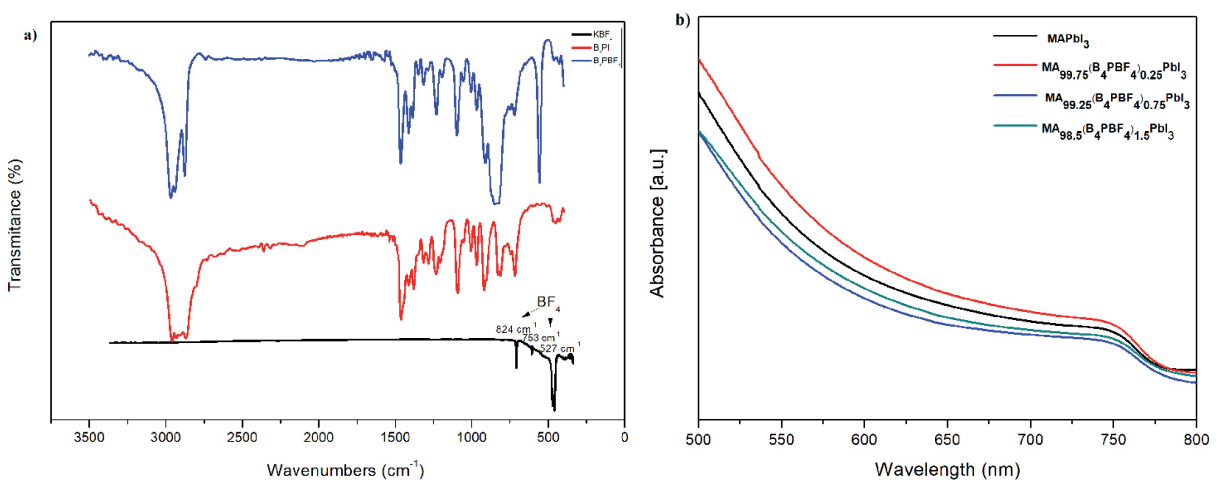

Fig 2. a) Espectros de $\mathrm{LI}_{-} \mathrm{B}_{4} \mathrm{PBF}_{4}$. Espectro azul $\mathrm{B}_{4} \mathrm{PBF}_{4}$, espectro rojo $\mathrm{B}_{4} \mathrm{PI}$ y espectro negro $\mathrm{BF}_{4}$; b) Espectros UV-Vis de película $\mathrm{B}_{4} \mathrm{PBF}_{4}$ diversos intervalos $(0 \%, 0.25 \%, 0.75 \%$ y $1.5 \%)$.

espectros de absorción en $750 \mathrm{~nm}$. Las películas con $\mathrm{B}_{4} \mathrm{PBF}_{4}$ muestran un ligero desplazamiento en comparación del espectro de la película sin aditivo, según estudios previos de diversos autores como Zhang et al, Liao et al. Este ligero desplazamiento indica la presencia de una estructura 2D-3D en la película. ${ }^{14-16}$ Esto se confirma más adelante con el análisis de la técnica de difracción de rayos $\mathrm{X}(\mathrm{XRD})$. En la figura 3a, muestra el análisis de $\mathrm{H}^{1} \mathrm{RMN}$ del precursor $\mathrm{B}_{4} \mathrm{PI}$ y el líquido iónico $\mathrm{B}_{4} \mathrm{PBF}_{4}$ los resultados son muy similares debido a la similitud de sus grupos funcionales, sin embargo, algunas diferencias permiten establecer la obtención de $\mathrm{B}_{4} \mathrm{PBF}_{4}$. El espectro $\mathrm{B}_{4} \mathrm{PI}$ muestra espectros característicos de los protones asociados con las cadenas de alquilo $\delta=0.96$ a $2.54 \mathrm{ppm}$. En cambio, el espectro rojo cuando se incorpora el anión $\mathrm{BF}_{4}$, presenta desplazamientos en cada señal. El espectro $\mathrm{P}^{31} \mathrm{RMN}$, del precursor $\mathrm{B}_{4} \mathrm{PI}$ muestra una sola señal en $\delta=32.7 \mathrm{ppm}$, este indica una transformación trifenilfosfina. Lo mismo sucede con el líquido iónico $\mathrm{B}_{4} \mathrm{PBF}_{4}$, a pesar de ello presenta un desplazamiento de $\delta=32.7$ a $\delta=33.1 \mathrm{ppm}$. Esto se puede atribuir a la presencia del anión $\mathrm{BF}_{4}$. Mediante $\mathrm{B}^{11}$ RMN del líquido iónico $\mathrm{B}_{4} \mathrm{PBF}_{4}$, se pudo comprobar la presencia de boro con hibridación $\mathrm{sp}^{3}$ presente en $\mathrm{BF}_{4}$ con una geometría tetraédrica, esto comparado con lo reportado en la literatura que reportan un desplazamiento químico en el rango de $\delta-1.0$ ppm. ${ }^{17}$

Los resultados más notables que se han obtenido por el momento en esta investigación, son la obtención de nuevas películas de perovskitas híbridas con $\mathrm{B}_{4} \mathrm{PBF}_{4}$, se han observado resultados prometedores de estabilidad frente a la humedad. El proceso de ingeniería de aditivo mejoro significativamente la calidad de las películas, el análisis por microscopia óptica (figura 4) muestra la superficie de las películas delgadas. Se puede examinar la formación de diferentes morfologías para los compuestos de películas sintetizadas $\left(\mathrm{MAPbI}_{3}\right.$, $\mathrm{MA}_{99.75}\left(\mathrm{~B}_{4} \mathrm{PBF}_{4}\right)_{0.25} \mathrm{PbI}_{3}, \mathrm{MA}_{99.25}\left(\mathrm{~B}_{4} \mathrm{PBF}_{4}\right)_{0.75} \mathrm{Pbl}_{3}$ y $\left.\mathrm{MA}_{98.5}\left(\mathrm{~B}_{4} \mathrm{PBF}_{4}\right)_{1.5} \mathrm{Pbl}_{3}\right)$. Las películas con aditivo $0.25 \%$ y $0.75 \%$ mostraron una mayor uniformidad en la morfología de las películas (figura $4 \mathrm{~b}$ y $4 \mathrm{c}$ ), esto podría ayudar al transporte de carga y una menor recombinación. Esto se confirma con estudios previos del grupo de trabajo de Sánchez et al., donde utilizan el yoduro de tetrabutil fosfonio $\left(\mathrm{B}_{4} \mathrm{PI}\right)$ obteniendo eficiencias del $15 \%$. ${ }^{16}$ Por lo que en este trabajo se propone como catión orgánico en conjunto con el anión hidrófobo tetrafluoroborato $\left(\mathrm{BF}_{4}\right)$ para la formación de un nuevo LI, como una alternativa para resolver los 

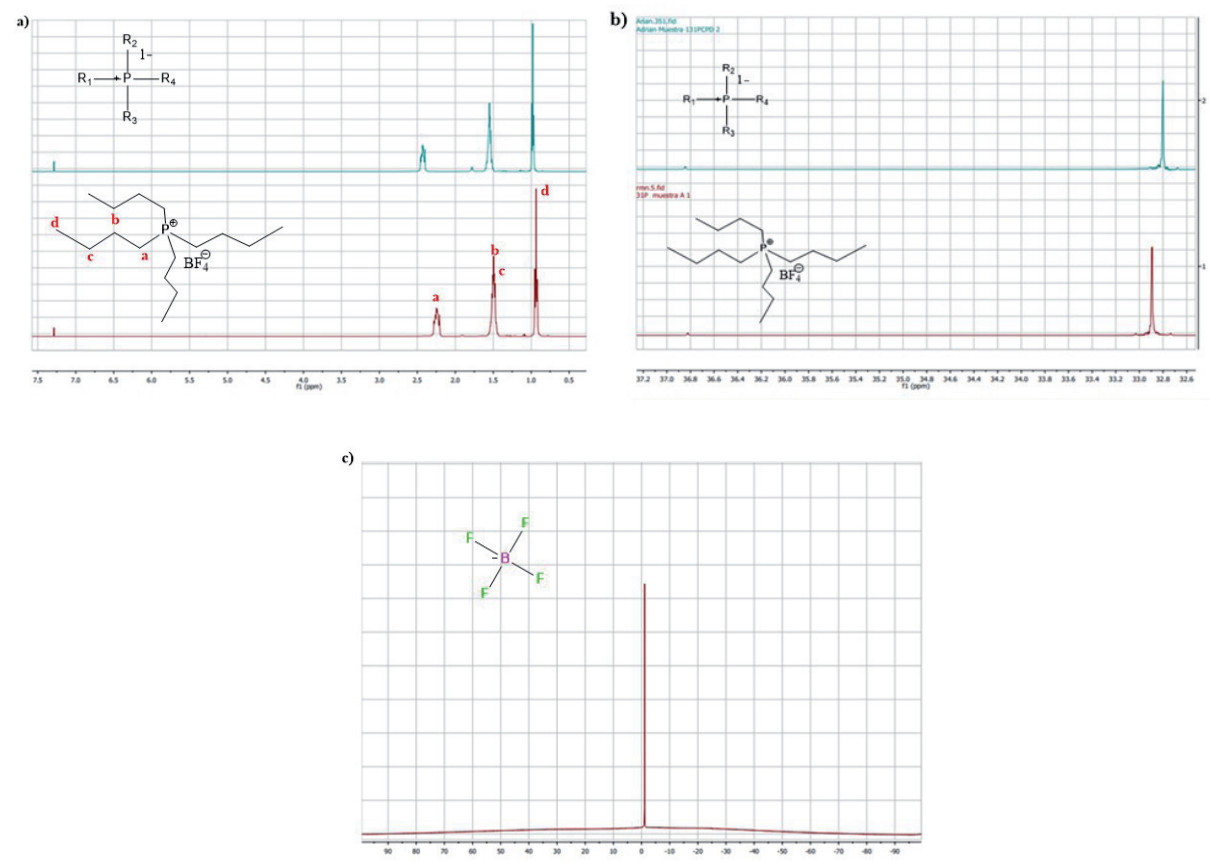

Fig. 3. a) RMN H1 espectro verde $B_{4} P I$, espectro rojo $B_{4} P B F_{4}$, b) RMN $P^{31}$ espectro verde $\mathrm{B}_{4} \mathrm{Pl}$, espectro rojo $\mathrm{B}_{4} \mathrm{PBF}_{4}$ y $\mathrm{c}$ ) $\mathrm{RMN} \mathrm{B}^{11}$ de $\mathrm{B}_{4} \mathrm{PBF}_{4}$.
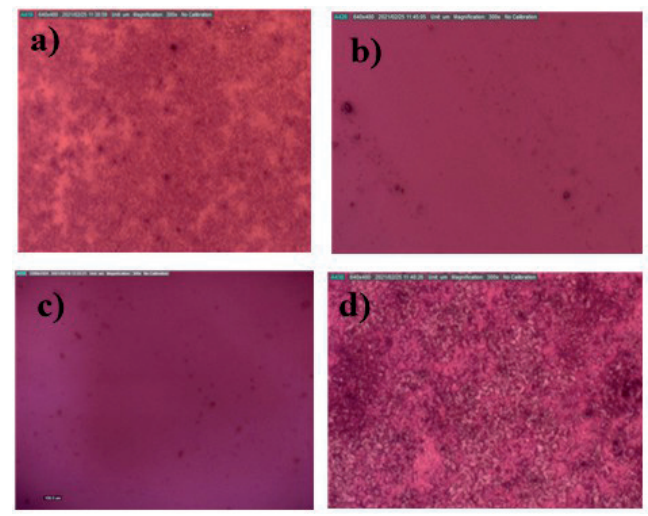

e)

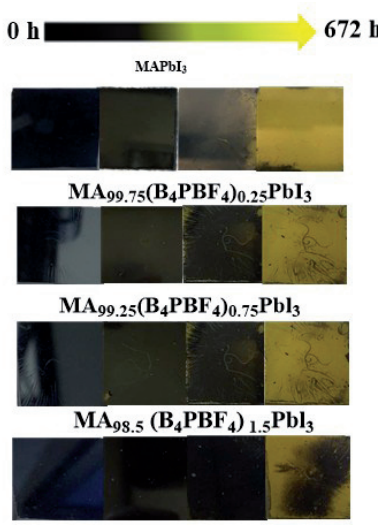

Fig. 4. Micrografías (magnificación: $300 \mathrm{X}$ ) de las películas de $M A \mathrm{Pbl}_{3} \mathrm{~B}_{4} \mathrm{PBF}_{4}$ depositadas a partir de las soluciones precursoras procesadas en diferentes intervalos, a) $\mathrm{MAPbl}_{3}$, b) $\left.\mathrm{MA}_{99.75}\left(\mathrm{~B}_{4} \mathrm{PBF}_{4}\right)_{0.25} \mathrm{Pbl}_{3}, \mathrm{C}\right) \mathrm{MA}_{99.25}\left(\mathrm{~B}_{4} \mathrm{PBF}_{4}\right)_{0.75} \mathrm{Pbl}_{3}$ y d) $\mathrm{MA}_{98.5}\left(\mathrm{~B}_{4} \mathrm{PBF}_{4}\right)_{1.5} \mathrm{Pbl}_{3} ;$ e) monitoreo de la degradación de las películas $\mathrm{MAPbl}_{3} \mathrm{~B}_{4} \mathrm{PBF}_{4}$, expuestas a una humedad relativa (HR) controlada (35\%-40\%).

problemas asociados a la estabilidad de las perovskitas a base de $\mathrm{MAPbl}_{3}$. Los LI hidrofóbicos a base de fosfonio muestran buenas propiedades para su aplicación en CSP, como es su estabilidad térmica, baja presión de vapor, amplio ensamblaje con anión-catión orgánicos e inorgánicos para la formación de LIs posibles. ${ }^{18,19}$, los cationes de fosfonio pueden interactuar con el plano cristalográfico de la perovskita terminando en la esquina que comporte octaedros de haluro metálico y forman estructura orgánico-inorgánico en capas. ${ }^{11,20,21}$ En la figura $4 \mathrm{e}$, se documentó la degradación de las películas expuestas a una humedad relativa (HR) 

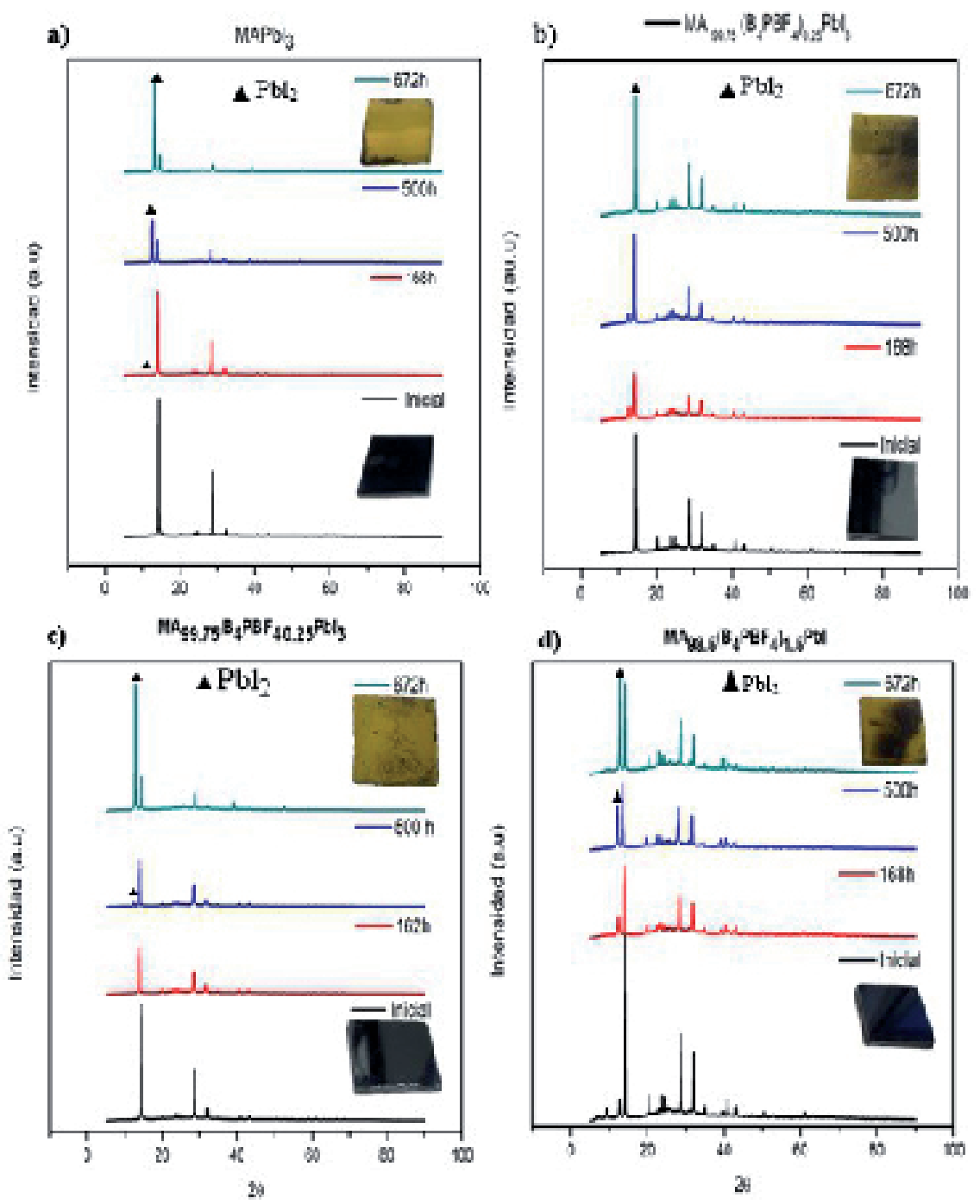

Fig. 5. Resultados de las muestras de estabilidad mediante XRD, para las películas sintetizadas $\mathrm{MAPbl}_{3 .} \mathrm{B}_{4} \mathrm{PBF}_{4}$; a) $\mathrm{MAPbl}_{3}$, b) $\left.\mathrm{MA}_{99.75}\left(\mathrm{~B}_{4} \mathrm{PBF}_{4}\right)_{0.25} \mathrm{Pbl}_{3}, \mathrm{C}\right) \mathrm{MA}_{99.25}\left(\mathrm{~B}_{4} \mathrm{PBF}_{4}\right)_{0.75} \mathrm{Pbl}_{3}$ y d) $\mathrm{MA}_{8.5}\left(\mathrm{~B}_{4} \mathrm{PBF}_{4}\right)_{1.5} \mathrm{Pbl}_{3}$.

controlada del $35 \%-40 \%$. Los resultados en cuanto a estabilidad al ambiente son para las películas con aditivo muestran una menor degradación hasta las 500 horas principalmente para $\mathrm{MA}_{98.5}\left(\mathrm{~B}_{4} \mathrm{PBF}_{4}\right)_{1.5} \mathrm{Pbl}_{3}$., sin demostrar algún tipo de deterioro. Se podría decir que debido a la cadena del alquil y del anión hidrófobo, ha logrado mantener por periodos más largos la estabilidad, comparando con las películas de control. El análisis de XRD nos confirma la obtención de estructuras tipo perovskita, muestra el patrón $\mathrm{MAPbI}_{3}$ característico observado en $14.1^{\circ}, 20^{\circ}$, $23.5^{\circ}, 24.5^{\circ}, 28.5^{\circ}, 31.9^{\circ}, 40.6^{\circ}$ y $43.2^{\circ}$ que corresponden a los planos $\left(\begin{array}{lll}1 & 1 & 0\end{array}\right),\left(\begin{array}{ll}1 & 1\end{array}\right.$ 2), (2 1 1), (2 02 ), (2 20 0), (3 10), (2 24 4), (3 14 4), respectivamente. ${ }^{22-25}$ Las nuevas perovskitas híbridas también se analizaron periódicamente mediante XRD, para examinar como afecta la humedad su estructura cristalina. Como características de la degradación del material se presenta picos asociados al $\mathrm{Pbl}_{2}$ y cationes de $\mathrm{MA}^{+}$. Los difractogramas exhiben la evolución de la degradación de cada película (figura 5). Al principio del análisis la presencia del pico característico 
del $\mathrm{Pbl}_{2}\left(12.5^{\circ}\right)$, no se observa. Con el paso del tiempo de exposición, el pico comienza aparecer, en la película de $\mathrm{MAPbl}_{3}$ sin aditivo comienza aparecer a las 162 horas, mientras que la película con el LI-B $\mathrm{PBF}_{4}$, comienza a degradarse a tiempos más largos. Los resultados muestran que la incorporación del $1.5 \%$ de $\mathrm{LI}-\mathrm{B}_{4} \mathrm{PBF}_{4}$, mejoró la estabilidad de las películas $\mathrm{MAPbI}_{3}-\mathrm{B}_{4} \mathrm{PBF}_{4}$ en un mayor porcentaje. El mejoramiento de la estabilidad se podría deber a la interacción de los iones de fosforo, $\mathrm{Pbl}_{2} \mathrm{y}$ el anión hidrofóbico $\mathrm{BF}_{4}$. En conjunto con la cadena de alquilo en las capas de $\mathrm{MAPbl}_{3}$ promueve la formación de una estructura en la superficie más homogénea (figura $6 \mathrm{~b}, \mathrm{c}$ y d). ${ }^{16,26}$ Además aparece un pico en las películas dopadas en un ángulo de $7.8^{\circ}$ que puede asignarse a un plano (020) perteneciente a una estructura $2 \mathrm{D}$. ${ }^{27,28} \mathrm{La}$ aparición de dicha estructura también se confirmada por los análisis de UV-Vis, este pico se ve mayormente reflejado en las películas dopadas con el porcentaje de $1.5 \%$ de aditivo, se podría significar que el LI- $\mathrm{B}_{4} \mathrm{PBF}_{4}$ se encuentra entre capas de $\mathrm{MAPbl}_{3}$ y actúa como una barrera para bloquear la degradación causada por la humedad en las películas.

\section{CONCLUSIONES}

Por medio de la síntesis de este nuevo aditivo $\mathrm{B}_{4} \mathrm{PBF}_{4}$ y su incorporación a las películas de $\mathrm{MAPbl}_{3}$, se logró documentar periódicamente su estabilidad a largo plazo. Se encontró que las películas dopadas en condiciones ambientales con HR presentan una estabilidad por tiempos mayores a 162 horas, que es donde se comenzó a degradar las películas no dopadas. Cabe destacar que el mejor porcentaje $(1.5 \%)$ de dopaje presento resultados prometedores ante la inestabilidad a las 500 horas, después de las cuales no se presentó ningún tipo de degradación. Sin embargo, se está trabajando en la aplicación fotovoltaica de este nuevo aditivo en las CSP, se espera obtener resultados ventajosos debido al cambio notable de morfología en la superficie de las películas que proporciona este LI, mejore el transporte de carga y disminuya la recombinación de los dispositivos.

\section{AGRADECIMIENTOS}

Los autores agradecen a la Universidad Autónoma de Nuevo León, SENERCONACyT por apoyar financieramente esta investigación con el proyecto 256766 y proyecto CONACyTFC-2015-2-1252.

\section{REFERENCIAS BIBLIOGRÁFICAS}

1. Kim JY, Lee JW, Jung HS, Shin H, Park NG. High-Efficiency Perovskite Solar Cells. Chem Rev. 2020;120(15):7867-7918. doi:10.1021/acs. chemrev.0c00107

2. Dieter W. CH3NH3PbX3, ein $\mathrm{Pb}(\mathrm{II})$-System mit kubischer Perowskitstruktur /CH3NH3PbX3 a Pb (II) (-System with Cubic Perovskite Structure. Zeitschrift für Naturforsch B. 1978;33(12):1443-1445. https://www.degruyter.com/view/ journals/znb/33/12/article-p1443.xml

3. Mitzi DB, Wang S, Feild CA, Chess CA, Guloy AM. Conducting layered organic-inorganic halides containing $\langle 110\rangle$-oriented perovskite sheets. Science (80- ). 1995;267(5203):1473-1476. doi:10.1126/science.267.5203.1473 
Ingenierías de aditivos en celdas solares tipo perovskita / Ana Itzel Santiago Mustafat, et al.

4. Son DY, Kim SG, Seo JY, et al. Universal Approach toward HysteresisFree Perovskite Solar Cell via Defect Engineering. J Am Chem Soc. 2018;140(4):1358-1364. doi:10.1021/jacs.7b10430

5. Zhang M, Bing J, Cho Y, et al. Synergistic effect of potassium and iodine from potassium triiodide complex additive on gas-quenched perovskite solar cells. Nano Energy. 2019;63. doi:10.1016/j.nanoen.2019.06.049

6. Yang J, Chen S, Xu J, et al. A review on improving the quality of Perovskite Films in Perovskite Solar Cells via the weak forces induced by additives. Appl Sci. 2019;9(20). doi:10.3390/app9204393

7. Shahiduzzaman M, Yamamoto K, Furumoto Y, Kuwabara T, Takahashi $\mathrm{K}$, Taima $\mathrm{T}$. Ionic liquid-assisted growth of methylammonium lead iodide spherical nanoparticles by a simple spin-coating method and photovoltaic properties of perovskite solar cells. RSC Adv. 2015;5(95):77495-77500. doi:10.1039/c5ra08102e

8. Deng X, Xie L, Wang S, et al. Ionic liquids engineering for high-efficiency and stable perovskite solar cells. Chem Eng J. 2020;398:125594. doi:10.1016/ j.cej.2020.125594

9. Du J, Wang Y, Zhang Y, et al. Ionic Liquid-Assisted Improvements in the Thermal Stability of CH3NH3PbI3 Perovskite Photovoltaics. Phys Status Solidi - Rapid Res Lett. 2018;12(8):1-6. doi:10.1002/pssr.201800130

10.Hu Z, Zheng N, Dong S, et al. Phosphonium conjugated polyelectrolytes as interface materials for efficient polymer solar cells. Org Electron. 2018;57:151157. doi:10.1016/j.orgel.2018.03.006

11. Sun C, Xue Q, Hu Z, et al. Phosphonium Halides as Both Processing Additives and Interfacial Modifiers for High Performance Planar-Heterojunction Perovskite Solar Cells. Small. 2015;11(27):3344-3350. doi:10.1002/ smll.201403344

12.Zhang F, Zhu K. Additive Engineering for Efficient and Stable Perovskite Solar Cells. Adv Energy Mater. 2020;10(13):1-26. doi:10.1002/aenm.201902579

13.Wu Q, Zhou W, Liu Q, et al. Solution-Processable Ionic Liquid as an Independent or Modifying Electron Transport Layer for High-Efficiency Perovskite Solar Cells. ACS Appl Mater Interfaces. 2016;8(50):34464-34473. doi:10.1021/acsami.6b12683

14.Zhang W, Lei X, Liu J, et al. Efficient Charge Collection Promoted by Interface Passivation Using Amino Acid Toward High Performance Perovskite Solar Cells. Phys Status Solidi - Rapid Res Lett. 2019;13(2):1-6. doi:10.1002/ pssr.201800505

15.Liao Y, Liu H, Zhou W, et al. Highly Oriented Low-Dimensional Tin Halide Perovskites with Enhanced Stability and Photovoltaic Performance. J Am Chem Soc. 2017;139(19):6693-6699. doi:10.1021/jacs.7b01815

16.Gonzalez-Juarez E, Valadez-Villalobos K, Garcia-Gutierrez DF, GarciaGutierrez DI, Roa AE, Sanchez E. Study on photovoltaic stability and performance by incorporating tetrabutyl phosphonium iodide into the active layer of a perovskite type photovoltaic cell. RSC Adv. 2020;10(52):3157531585. doi:10.1039/d0ra04630b 
Ingenierías de aditivos en celdas solares tipo perovskita / Ana Itzel Santiago Mustafat, et al.

17. Saihara K, Yoshimura Y, Fujimoto H, Shimizu A. Detrimental effect of glass sample tubes on investigations of BF4--based room temperature ionic liquid-water mixtures. J Mol Liq. 2016;219:493-496. doi:10.1016/ j.molliq.2016.03.036

18.Ullah Z, Azmi Bustam M, Man Z, Khan AS. Phosphonium-based ionic liquids and their application in separation of dye from aqueous solution. ARPN J Eng Appl Sci. 2016;11(3):1653-1659.

19. Mostafa MF, Atallah AS, Elessawi M. Preparation and characterization of a new series of perovskite-like structures showing evidence of structural transitions: (Methyltriphenylphosphonium)2 BX4, B = Mn, $\mathrm{Co}, \mathrm{Cu}$, and $\mathrm{Hg}$, and $\mathrm{X}=\mathrm{Cl} / \mathrm{I}$. Phase Transitions. 1998;64(4):215-227. doi:10.1080/0141159 9808208000

20.Kagan CR, Mitzi DB, Dimitrakopoulos CD. Organic-inorganic hybrid materials as semiconducting channels in thin- film field-effect transistors. Science (80-). 1999;286(5441):945-947. doi:10.1126/science.286.5441.945

21. Chondroudis K, Mitzi DB. Electroluminescence from an organic-inorganic perovskite incorporating a quaterthiophene dye within lead halide perovskite layers. Chem Mater. 1999;11(11):3028-3030. doi:10.1021/cm990561t

22.Wang C, Zhang C, Huang Y, et al. Degradation behavior of planar heterojunction CH3NH3PbI3 perovskite solar cells. Synth Met. 2017;227:4351. doi:10.1016/j.synthmet.2017.02.022

23.Xia Z, Chai G, Wang Y, Zhou H. Uniform perovskite photovoltaic thin films via ultrasonic spray assisted deposition method. 2015 IEEE 42nd Photovolt Spec Conf PVSC 2015. Published online 2015:1-4. doi:10.1109/ PVSC.2015.7355719

24.Chen LC, Weng CY. Optoelectronic Properties of MAPbI3 Perovskite/ Titanium Dioxide Heterostructures on Porous Silicon Substrates for Cyan Sensor Applications. Nanoscale Res Lett. 2015;10(1):3-7. doi:10.1186/ s11671-015-1114-x

25.Rajendra Kumar G, Dennyson Savariraj A, Karthick SN, et al. Phase transition kinetics and surface binding states of methylammonium lead iodide perovskite. Phys Chem Chem Phys. 2016;18(10):7284-7292. doi:10.1039/c5cp06232b

26.Jin S, Wei Y, Rong B, et al. Improving perovskite solar cells photovoltaic performance using tetrabutylammonium salt as additive. J Power Sources. 2020;450(December 2019):227623. doi:10.1016/j.jpowsour.2019.227623

27. Wei Y, Chu H, Tian Y, et al. Reverse-Graded 2D Ruddlesden-Popper Perovskites for Efficient Air-Stable Solar Cells. Adv Energy Mater. 2019;9(21):1-9. doi:10.1002/aenm.201900612

28. Mao L, Ke W, Pedesseau L, et al. Hybrid Dion-Jacobson 2D Lead Iodide Perovskites. J Am Chem Soc. 2018;140(10):3775-3783. doi:10.1021/ jacs. 8 b00542 